\title{
Meaningful work
}

Jitske M. C. Both-Nwabuwe, Maria T. M. Dijkstra, Bianca Beersma

\section{Source}

Jitske M. C. Both-Nwabuwe, Maria T. M. Dijkstra, Bianca Beersma. (2017). Sweeping the Floor or Putting a Man on the Moon: How to Define and Measure Meaningful Work. Front. Psychol., vol. 8. doi:10.3389/fpsyg.2017.01658.

meaningful work is the subjective experience of existential significance resulting from the fit between the individual and work. 